\section{FRI0257 CHANGES IN SKELETAL MUSCLES IN PATIENTS WITH KNEE JOINT REPLACEMENT}

${ }^{1} \mathrm{E}$ Neuen-Jacob, ${ }^{2} \mathrm{M}$ Meinhardt, ${ }^{1} \mathrm{M}$ Egl, ${ }^{3} \mathrm{~B}$ Fink. 'Department of Neuropathology, University Hospital Düsseldorf, Düsseldorf; ${ }^{2}$ Department of Pathology, Technical University, Dresden; ${ }^{3}$ Department of Orthopedics, University Hospital Eppendorf, Hamburg, Germany

\subsection{6/annrheumdis-2001.578}

Background Knee joint replacement is used as a routine surgical procedure in the treatment of rheumatoid diseases or arthrosis. However, systematical studies on the affected muscles are rare.

Objectives In the present systematic study we analysed the pathological changes in the vastus medialis muscles in 108 patients with rheumatoid arthritis and gonarthrosis who underwent knee joint replacement. The aim of the current study was to examine possible differences in both entities with respect to kind and degree of muscle changes and to the state of innervation/denervation which might be important for the therapeutic effect of the arthroplasty.

Methods Serial cross-cut cryostat sections were stained by H\&E and Gomori's trichrome and submitted to routine histochemical preparations including NADH/TR, ATPase after preincubation at pH 4.6 and 9.4, oil-red O, periodic acid Schiff and acid phosphatase. Immunohistochemistry was performed using commercially available monoclonal antibodies (Mab) against neonatal myosin and neural cell adhesion molecule CD56 for the identification of regenerating fibres. In addition, the Mab against HLA$\mathrm{ABC}$ was applied in order to detect the up-regulation of $\mathrm{MHC}$ Class I antigen on the muscle fibres.

Results Lipomatosis was striking in the majority of both groups. Regenerating fibres also were detectable in both entities. As a trend, patients with gonarthrosis mainly showed atrophy of type 2 fibres indicating inactivity due to muscle or joint pain. Patients with rheumatoid arthritis commonly revealed atrophy of both type 1 and 2 fibres and fibre type grouping consistent with neurogenic muscular atrophy indicating a systemic disease. Vasculitis was only occasionally detected. In rheumatoid arthritis, however, up-regulation of MHC Class I antigen was regularly seen reflecting the underlying autoimmune disease. A small percentage of cases showed corticoid myopathy with atrophy of type 2 fibres in combination with increased lipid content of type 1 fibres. Conclusion

\section{OP0129 GREATER TROCHANTERIC PAIN SYNDROME. ULTRASOUND AND CLINICAL CORRELATIONS}

C Orellana, A Ponce, P Barceló. Rheumatology Unit, URB, Barcelona, Spain

\subsection{6/annrheumdis-2001.579}

Background Greater trochanteric pain syndrome consists of a common clinical syndrome including ?classical? trochanteric bursitis but also gluteus medius pathology, with main clinical features being pain and tenderness over the lateral aspect of hip.

Objectives To assess the prevalence of trochanteric bursitis and gluteus medius tendonitis by ultrasound examination, together or in isolation, in patients with greater trochanteric pain syndrome and to investigate whether different clinical features could be related to ultrasound findings.

Methods $36(34 \mathrm{~F} / 2 \mathrm{M})$ patients with greater trochanteric pain syndrome were studied retrospectively. A coxofemoral or lumbar origin of pain was ruled out in all these patients. The following variables were used: age, disease duration, presence of obesity or leg length inequity, pain type (use-related/at rest) and location (trochanter, buttock or both), Trendelemburg sign and ressted hip abduction or internal rotation, and also the existence of calcifications or significant osteoarthritis changes on X-ray examination.

Results Acute or chronic signs of gluteus medius tendinitis were found in 22 patients (61\%), and inflammatory changes suggesting both trochanteric bursitis and gluteus medius pathology were detected in $12(33 \%)$; isolated trochanteric bursitis was found in only 2 cases $(6 \%)$. Disease duration was longer in patients with inflammatory changes at both locations $(14 \mathrm{mo}$ vs $6 \mathrm{mo}, \mathrm{p}=$ 0.018 ), but there were no differences relating age. No significant clinical features allowed differentiation of the ultrasound findings, although a tendency towards pain location defining the underlying pathology could be observed ( $p=0.062$ for trochanteric pain, $\mathrm{p}=0.09$ for buttock pain). Patients showing inflammatory changes in both gluteus and trochanteric bursa on ultrasound tended to be more obese than those without bursitis $(\mathrm{p}=0.073)$ and calcications on X-ray tended to be found more frequently in those with only gluteus medius tendonitis $(\mathrm{p}=$ 0.082).

Conclusion Isolated trochanteric bursitis was rarely found on ultrasound examination in patients with greater trochanteric pain syndrome, but gluteus medius tendon inflammatory changes could be found in the vast majority of cases, most of them without associated trochanteric bursitis. No reliable clinical features could be used to distinguish between ultrasound changes.

\section{OP0130 UNIQUE ULTRASONOGRAPHIC FINDINGS IN PATIENTS WITH THE HEREDITARY INCLUSION BODY MYOPATHY}

${ }^{1} \mathrm{GM}$ Garofalo, ${ }^{2} \mathrm{R}$ Adler, ${ }^{3} \mathrm{D}$ Darvish, ${ }^{4} \mathrm{~S}$ Rafii, ${ }^{5} \mathrm{~S}$ Paget, ${ }^{5} \mathrm{~L}$ Kagen. ${ }^{1}$ Istituto Di Clinica Medica E Reumatologia, Ospedale Garibaldi, Catania, Italia; ${ }^{2}$ Radiology Department, Hospital for Special Surgery, New York, USA; ${ }^{3}$ Cedars Sinai Medical Center, UCLA, Los Angeles, USA; ${ }^{4}$ Hematology, Weill Medical College Cornell University, New York, USA; ${ }^{5}$ Rheumatology Division, Hospital for Special Surgery, New York, USA

\subsection{6/annrheumdis-2001.580}

Background Hereditary Inclusion Body Myopathy (HIBM) is an autosomal recessive disorder characterised histologically by the presence of beta amyloid fibrils in myofibers and clinically by muscle weakness and atrophy with quadriceps sparing. This disorder is seen in different ethnic groups including members of Iranian Jewish families where it has been mapped to chromosome 9p1-q1. The onset is usually in the 2 nd to 3 rd decade of life, with slow progression leading to severe involvement of multiple muscle groups, ultimately resulting in immobilisation.

Objectives To demonstrate the facility of the use of grey scale and power Doppler sonography as a non-invasive diagnostic procedure in the evaluation of HIBM.

Methods We studied 4 Iranian Jewish patients diagnosed with HIBM. Ultrasonographic examinations of the quadriceps femoris and hamstring muscle groups were carried out in these patients. Echogenicity, as determined by grey scale assessment, was used to detect disordered muscle structure and atrophy, and power Doppler sonography was used to assess vascularity.

Results The initial symptom in all was "footdrop" due to anterior tibial muscle weakness. Two patients with disease duration of seven years developed pelvic girdle and shoulder girdle weakness. Two patients with twenty-eight years of disease were restricted to wheelchairs with severe myopathy of the upper and lower extremities. All had significant family histories consistent with HIBM. An unusual and unique pattern of central atrophy of muscle with peripheral sparing was observed in all four 
patients. Vascularity was markedly reduced in the affected areas with relatively increased blood flow in the peripherally-spared areas. We referred to this as a "fried-egg" or "bull's eye" appearance.

Conclusion Our study demonstrates the use of both grey scale and power Doppler sonography as a helpful non-invasive procedure in the evaluation of HIBM. Their ability to define a unique centrifugal, "fried-egg" myopatic abnormality may be diagnostic for this disorder.

\section{OP0131 CHLOROQUINE MYOPATHY: PREVALENCE AND CLINICAL FEATURES (PRELIMINARY DATA)}

${ }^{1} \mathrm{E}$ Casado, ${ }^{1} \mathrm{M}$ Larrosa, ${ }^{1 \mathrm{~J}} \mathrm{Gratacos},{ }^{2} \mathrm{~J}$ Font, ${ }^{3} \mathrm{JM}$ Martinez, ${ }^{2} \mathrm{C}$ Tolosa. ${ }^{1}$ Rheumatology; ${ }^{2}$ Medicine; ${ }^{3}$ Neurology, Corporacio Sanitaria Parc Tauli. University Hospital of Sabadell, Sabadell, Spain

10.1136/annrheumdis-2001.581

\section{Background}

Objectives To analyse the prevalence and clinical features of chloroquine myopathy in a cohort of out-patients in a Rheumatology Unit.

Methods Prospective study of 119 patients (84 female and 35 male), aged $57.8 \pm 13.7$, taking antimalarials (112 chloroquine, 7 hydroxychloroquine) for more than 6 months. They were evaluated during 2 years. The underlying disease was RA in 69, connective tissue disease in 22, palindromic rheumatism in 14 , spondyloarthropathy in 7 and other rheumatic disease in 7 . Muscle enzymes were determined in all patients and an electromyography (EMG) was practised in patients with repeated increased determinations. Patients with altered EMG were biopsied.

Results Laboratory: 18 patients (15\%) had a repeated increase of muscle enzymes: LDH in 17 patients, CPK in 5 and aldolase in 1 patient. EMG: practised in 13 patients, showed a muscular involvement in 6 . Two patients are pending and 3 were dead before the exam. Muscle biopsy: 6/7 patients had a chloroquine myopathy (optic and electronic microscopy); 1/7 patients had a steroid myopathy. Two patients (RA) with chloroquine myopathy had an associated inflammatory myositis. Clinical features: 6 patients with chloroquine myopathy (5 RA and 1 spondyloarthropathy) represents an accumalated prevalence (2 years) of $5 \%$. The duration of treatment was36 \pm 21 months (rest of the group $40 \pm 31$ ), with cumulative doses of $203 \pm 25 \mathrm{~g}$ (rest of the group $305 \pm 243 \mathrm{~g}$ ). All patients presented weakness and had other simultaneous muscle involvement factors (1 cervical myelopathy, 2 hypothyroidism, 3 hypovitaminosis D and 1 prolonged bedrest) at clinical diagnosis.

Conclusion Toxic chloroquine myopathy is not an excepcional event (accumulated prevalence 5\%). When an increase of LDH and/or CPK is present we should to rule out this complication. The presence of other factor with muscle involvement probably makes worse the weakness and gets better its detection.

\section{OP0132 MYOSITIS AND PREGNANCY}

CA Silva, DA Isenberg. Centre for Rheumatology, University College of London, London, UK

10.1136/annrheumdis-2001.582

Background Polymyositis and dermatomyositis are the most common form of idiopathic inflammatory myopathy, overall the female to male ratio is $2-3: 1$. The age at presentation is most often between 40 and 60 years. Pregnancies in these patients are uncommon, and the overall maternal and foetal risks are not well defined in patients with myositis.

Objectives We assessed the prevalence and outcome of pregnancy in 37 patients with dermatomyositis and polymyositis.

Methods 54 patients with myositis have been treated between 1976 and 2000 at the Bloomsbury Rheumatology Unit, University College/Middlesex Hospital. A prospective record of all such patients had been kept by one of us (DAI). All patients meet the Bohan and Peter Criteria for DM and PM. The presence of another autoimmune disease was noted as appropriate. Patients were reviewed on a regular basis in a specialist outpatient clinic, and for all patients basic demographic data, age at onset, treatment, number of pregnancies, activity of the disease during pregnancy were noted. For the purposes of this study all of the available 37 patients were examined by one of us (CS): 28 females and 9 males.

Results The female patients medium age was 44 years, with age at onset ranging from 16 to 64.16 have Dermatomyositis/Polymyositis (7- PM/9- DM) and 12 have myositis as part of an overlap syndrome (SLE (7 patients), 2 patients with RA, 2 with SS, and 2 with $\mathrm{Scl}$ ). Only 3 of our patients have been pregnant after the onset of the disease. One, with a poor control of her disease, had an abortion during the first trimester, and was also on treatment with MTX. The other two had the disease under control and 2 normal pregnancies with good foetal outcome was achieved. Both suspended immunosupressants during pregnancy and were able to decrease steroid dosage, without increasing disease activity.

Conclusion Pregnancy in myositis patients is a rare event. Pregnancies occuring in myositis patients with the disease under clinical remission can result in good foetal outcome. The activity of the disease remains stable. These results are consistent with the literature from recent years, and are probable due to better control of disease activity.

\section{Fibromyalgia}

\section{AB0166 THE REAL VALUE OF REHABILITATION IN THE MUSCULAR SYNDROMES OF THE CERVICAL SPINE}

EL Sidenco. Rehabilitation, University Hospital Bucharest, Bucharest, Romania

\subsection{6/annrheumdis-2001.583}

\section{Background}

Objectives We studied a group of 34 patients with algo-functional muscular-ligamentar cervical syndromes based on complex static disorders, diagnosticaly excluding cervical discal hernia. We propose a clinical -functional score for an unitary evaluation of these cases.

Methods We applied an initial kinetic programme for two weeks; we propose a complex of stretching-strengthening- coordination exercises for the cervical muscular contraction, supported by thermo-electro-massotherapy.

Results Efficiency of the initial kinetic programme was good, the following clinical parameters increased over $63,7 \%$.

The second stage of rehabilitation was applied a month later; we used a kinetic programme analogous to the first stage, for two weeks again, insisting on the strengthening- co-ordination exercises for the cervical muscles. The kinetic programme of the 